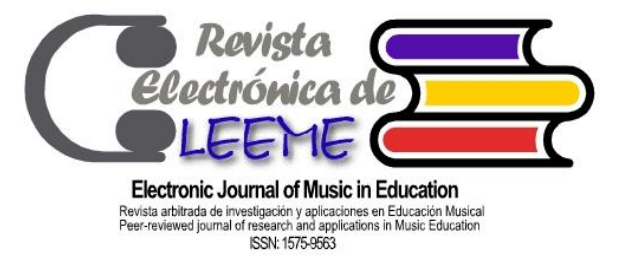

LUISA MOYA PASTOR

\title{
El aprendizaje del violín en el siglo XX: un estudio comparativo de los métodos
} Suzuki, Rolland y Colourstrings

Learning violin in the twentieth century: comparative study of Suzuki, Rolland and Colourstrings methods

Luisa Moya Pastor luiviolin@gmail.com Universidad Internacional de La Rioja

La Rioja, España

ORCID: https://orcid.org/0000-0002-6416-419X

doi: 10.7203/LEEME.41.10827

\section{Resumen}

El propósito de este trabajo es valorar en qué aspectos puede influir la aplicación de determinados métodos en el proceso de enseñanza-aprendizaje del violín. Para ello, en este artículo se plantea una revisión bibliográfica, así como un análisis comparativo de tres métodos de iniciación utilizados en la actualidad en el aprendizaje del violín: el método Rolland, el método Suzuki y el método Colourstrings. Se pretende buscar la validez de estos tratados identificando y comparando cuestiones referentes a la técnica y principios de cada uno, permitiendo ver las similitudes que comparten y los aspectos que las diferencian en las primeras etapas del aprendizaje de este instrumento. Las citadas metodologías poseen alternativas y recursos novedosos que, con una adecuada combinación, pueden ser adaptadas por el docente para utilizarse en la metodología actual.

Palabras clave: método; violín; pedagogía del violín; didáctica musical.

Abstract

The aim of this work is to assess aspects that influence the application of certain methods in the process of teaching and learning the violin. For this, we propose a bibliographic examination as a well as a comparative analysis of three methods for beginners currently used: Rolland method, Suzuki method, and Colourstrings method. We try to validate this process by identifying and comparing aspects regarding the technique and principles of each one, taking into account the similarities they share and the aspects in which they differ in their approaches to early stages of violin learning. Those methods offer original alternatives and resources that, when properly combined, can be adapted by teachers and added to current methodology.

Key words: methods; violin; violin teaching; music teaching.

(C) LUISA MOYA PASTOR. THE CONTENT OF THIS ARTICLE IS THE SOLE RESPONSIBILITY OF THE AUTHORS. THE REVISTA ELECTRÓNICA DE LEEME AND UNIVERSITAT DE VALĖNCIA ARE NOT LIABLE FOR ANY LEGAL ACTIONS THAT MAY ARISE INVOLVING THE ARTICLE'S CONTENT. REVISTA ELECTRÓNICA DE LEEME -LISTA ELECTRÓNICA EUROPEA DE MÚSICA EN LA EDUCACIÓN-HTTP://MUSICA.REDIRIS.ES.ISSN: 1575-9563 EDITORES: UNIVERSIDAD DE VALENCIA Y JESÚS TEJADA GIMÉNEZ. VISIBILIDAD DE ESTA REVISTA: EBSCO, CINDOC WORLDCAT, RESH, REDIB, RILM CORE JOURNALS, SUDOC, ULRICHS, ESTA REVISTA ESTÁ PUBLICADA CON EL APOYO INSTITUCIONAL DE REDIRIS-CONSEJO SUPERIOR DE INVESTIGACIONES CIENTIFICAS Y ES DE ACCESO LIBRE. CREATIVE COMMONS LICENSE 4.0 BY 

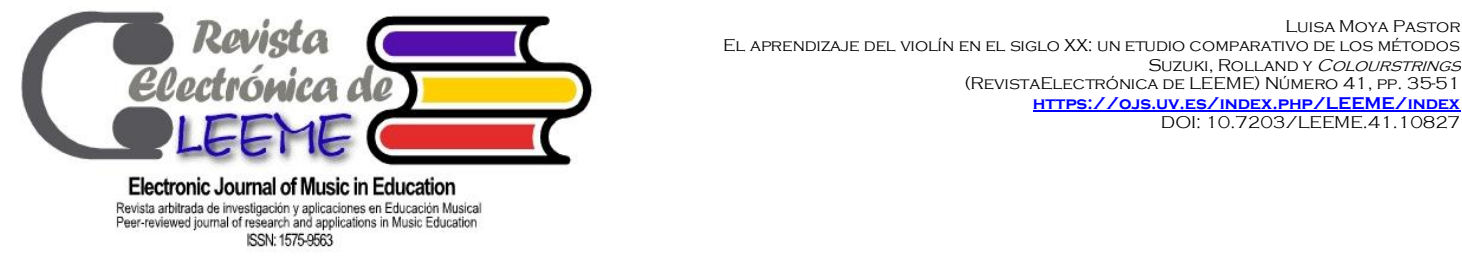

\section{Introducción}

El aprendizaje del violín se puede abordar de múltiples formas. Las primeras etapas de su aprendizaje son fundamentales para el desarrollo de hábitos, sensaciones y movimientos motores que acompañarán al estudiante a lo largo de su formación; por tanto, resulta imprescindible aplicar una pedagogía adecuada desde el inicio.

El propósito de esta investigación ha sido realizar una labor de selección y revisión bibliográfica sobre los Métodos Suzuki, Rolland y Colourstrings, que ha servido para realizar un análisis comparativo a partir de una selección de ítems fundamentales en el proceso de enseñanza/aprendizaje del violín. Este proceso se llevó a cabo estableciendo dos categorías: aspectos técnicos y no técnicos, comparándose su abordaje desde cada método. Este procedimiento ha resultado de utilidad para profundizar en ellos étodos, valorar el enfoque de los profesionales entrevistados y elaborar una propuesta pedagógica pertinente.

\section{Método}

\subsection{Contexto}

Las corrientes pedagógicas y musicales del siglo XX consideran la música parte de la realidad del niño, siendo éste el protagonista de su propio aprendizaje. En el ámbito del violín, se desarrollan nuevos enfoques metodológicos que apuestan por una enseñanza activa. Uno de estos enfoques es el Método Suzuki (1959), relevante por instaurar y trabajar aspectos referentes a la técnica o musicalidad. Entre sus características principales, destacan: la educación del talento, la importancia de un entorno favorable para el aprendizaje, y la creencia de que a través del aprendizaje se mejoran las capacidades y el estado anímico del niño.

Otro de los principales métodos, surgido en Estados Unidos, fue el Método Rolland (1974), resultado de una investigación realizada en la escuela pública americana sobre la pedagogía de las cuerdas. Su trascendencia se debe al enfoque orientado hacia un aprendizaje que cultiva la consciencia corporal: recibió grandes aportaciones de Alexander o de Hellebrant, que le ayudaron a valorar la importancia del cuerpo en la acción de tocar el violín. Fanelli (2011) cita los escritos de Rolland como fuente primaria de material para el estudio de la pedagogía de la cuerda, la fisiología y kinesiología del violinista. Entre sus características generales destacan: el desarrollo de la consciencia rítmica, el movimiento como eliminación de tensiones y la concepción de la técnica condensada en dos años.

Los hermanos Szilvay desarrollan el Método Colourstrings (1996). Su filosofía se basa en los valores de Kodály (1964), extrapolados al aprendizaje del violín: para ser un buen músico

(c) LUISA MOYA PASTOR. THE CONTENT OF THIS ARTICLE IS THE SOLE RESPONSIBILITY OF THE AUTHORS. THE REVISTA ELECTRÓNICA DE LEEME AND UNIVERSITAT DE VALĖNCIA ARE NOT LIABLE FOR ANY LEGAL ACTIONS THAT MAY ARISE INVOLVING THE ARTICLE'S CONTENT. REVISTA ELECTRÓNICA DE LEEME -LISTA ELECTRÓNICA EUROPEA DE MÚSICA EN LA EDUCACIÓN-HTTP://MUSICA.REDIRIS.ES.ISSN: 1575-9563 EDITORES: UNIVERSIDAD DE VALENCIA Y JESÚS TEJADA GIMÉNEZ. VISIBILIDAD DE ESTA REVISTA: EBSCO, CINDOC (CESIC), CITEFACTOR, COPAC, DIALNET, DICE (CSIC), DOAJ, E-REVISTAS (CSIC), EBSCO PREMIER, ERIH+, GALE CENGAGE LEARNING, IN-RECS, IRESIE, LATINDEX, MIAR, OCLC INVESTIGACIONES CIENTIFICAS Y ES DE ACCESO LIBRE. CREATIVE COMMONS LICENSE 4.0 BY 

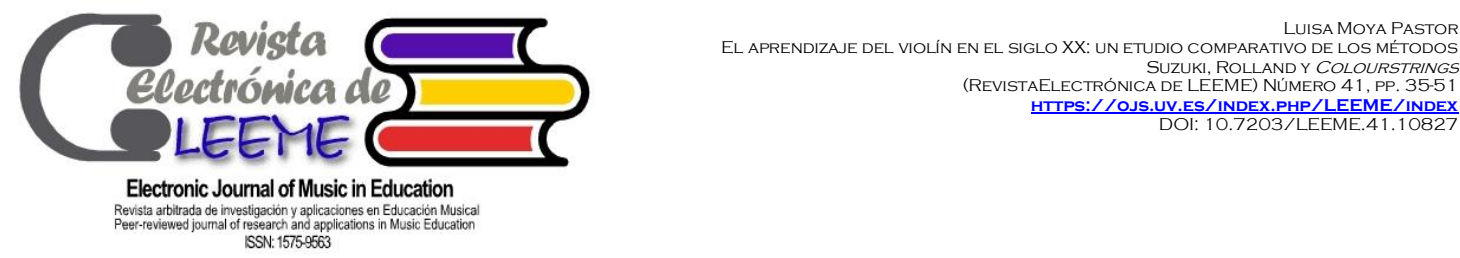

se deben formar por igual el oído, las emociones y la inteligencia. Entre sus principios generales, destacan: la enseñanza a través de los sentidos, la percepción visual por medio de colores y símbolos, el uso de representación rítmica no convencional y la importancia del canto y del solfeo relativo. Ruokonen, Juntunen y Ruismäki (2013) señalan que promueve el desarrollo integral del estudiante, donde la música se desarrolla como arte completo.

\subsection{Revisión bibliográfica}

En Suzuki (1969), se hallaron referencias para la "educación del talento". Respecto a éste se encontraron estudios como los de Starr (1976), que trata la capacidad innata del niño y constituye una guía para padres y profesorado. En Nelson (2011), se enfatiza la educación del talento, señalando su importancia para el alumnado, ya que no es una cualidad innata, sino educable y en desarrollo.

Se revisaron publicaciones de Rolland donde describió los principios fisiológicos del aprendizaje (Rolland, 1959), el estudio sobre el desarrollo del programa de cuerdas en la escuela pública americana (Rolland, 1979), Prelude to string playing (1971) fruto de la colaboración con Krolick y Rowell; y el resultado del programa de cuerdas y suma de las enseñanzas de Rolland (acompañado de 17 vídeos del proyecto desarrollado en la Universidad de Illinois) (Rolland, 1974). También, Fanelli (2011) señaló las influencias de Rolland para desarrollar su proyecto y dilucidar em qué aspectos básicos se basó. Nelson (2011) enfatiza la importancia del movimiento corporal a través del desarrollo de programas de cuerda en la escuela pública americana. Por su parte, Fray (1979) cita los escritos de Rolland como fuente primaria de material para el estudio de la pedagogía de la cuerda, la fisiología y kinesiología del violinista; y, Haun (1988) en su recopilación bibliográfica sobre más de 200 fuentes de pedagogía del violín destaca la relevancia de los escritos y talleres de Rolland.

En cuanto al Método Colourstrings, se hallaron estudios que señalan las influencias de Kodály a través de una adaptación de los ejercicios de la pedagogía Rolland mediante el uso de colores (Mitchell, 1994). Voima (2009) profundiza y destaca los contenidos de cada libro, por ejemplo, la lectura musical aparece a través del uso de grafías no convencionales, que supone un acercamiento progresivo y empático con el niño. En cuanto a ese uso de colores en la pedagogía instrumental, Kuo y Chuang (2013) han observado la raíz de vincular la música al color, consiguiendo fomentar la creatividad a través de esta visión cinestésica. También, se han encontrado artículos relacionados sobre el desarrollo integral del alumnado (Ruokonen, Juntunen y Ruismäki, 2013).

Entre otras fuentes de interés, se han encontrado: una comparación entre los métodos Suzuki, Rolland y Havas (Perkins, 1995); una comparación del método Suzuki con el método Rolland (Göktürk, 2011); y dos comparaciones entre las técnicas Suzuki, Rolland y Zweig

(C) LUISA MOYA PASTOR. THE CONTENT OF THIS ARTICLE IS THE SOLE RESPONSIBILITY OF THE AUTHORS. THE REVISTA ELECTRÓNICA DE LEEME AND UNIVERSITAT DE VALĖNCIA ARE NOT LIABLE FOR ANY LEGAL ACTIONS THAT MAY ARISE INVOLVING THE ARTICLE'S CONTENT. REVISTA ELECTRÓNICA DE LEEME -LISTA ELECTRÓNICA EUROPEA DE MÚSICA EN LA EDUCACIÓN-HTTP://MUSICA.REDIRIS.ES.ISSN: 1575-9563 EDITORES: UNIVERSIDAD DE VALENCIA Y JESÚS TEJADA GIMÉNEZ. VISIBILIDAD DE ESTA REVISTA: EBSCO, CINDOC (CESIC), CITEFACTOR, COPAC, DIALNET, DICE (CSIC), DOAJ, E-REVISTAS (CSIC), EBSCO PREMIER, ERIH+, GALE CENGAGE LEARNING, IN-RECS, IRESIE, LATINDEX, MIAR, OCLC INVESTIGACIONES CIENTIFICAS Y ES DE ACCESO LIBRE. CREATIVE COMMONS LICENSE 4.0 BY 

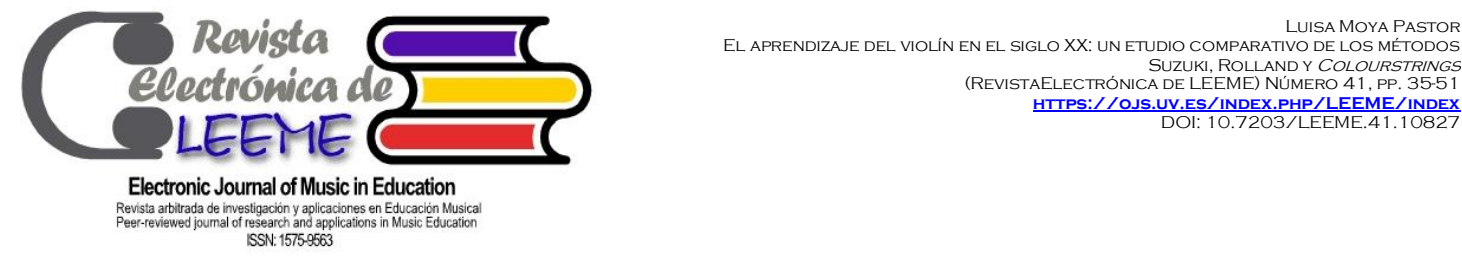

(Nelson, 2011; Soteras, 2013). La referencia más reciente se centra en la comparación al abordar la lectura musical entre las pedagogías Suzuki y Colourstrings (Jansen Van Vuuren, 2016), fundamental para esta investigación.

Como se puede observar, la investigación deriva hacia el estudio comparado para extrapolar los beneficios de cada uno a la práctica actual, simple o complementariamente. Resulta significativo que no todos los métodos seleccionados presentan una bibliografía especializada similar. El más difundido es el Método Suzuki, probablemente, por estar mundialmente extendido al contar con gran cantidad de asociaciones que organizan cursos de formación, jornadas pedagógicas y proyectos de revisión de partituras y grabaciones.

\section{Procedimiento}

\subsection{Plan de análisis}

Para llevar a cabo este estudio comparativo, se ha optado por destacar ciertos aspectos fundamentales del aprendizaje del violín contemplados en los métodos seleccionados. Para ello, se han distinguido dos categorías: aspectos técnicos y no técnicos.

Entre los aspectos técnicos, se han seleccionado herramientas básicas que deben desarrollarse para el aprendizaje. En este ámbito, se encuentran aspectos fisiológicos y psicológicos, dado que es el cerebro el que dirige los principales movimientos en el camino del aprendizaje. Y, entre los aspectos no técnicos, serían ciertos aspectos generales del aprendizaje que se han destacado por estar contemplados en los métodos abordados o por ser dignos de tener en cuenta para el aprendizaje del instrumento y del desarrollo artístico del músico.

Tabla 1. Aspectos analizados en los tres métodos

\begin{tabular}{cc}
\hline Aspectos técnicos & Aspectos no técnicos \\
\hline Postura corporal & Edad inicial \\
Afinación y sonido & Tipo de clase \\
Ritmo & Motivación \\
Mano izquierda & Lectura musical y memoria \\
Cambios de posición y armónicos & Repertorio \\
Vibrato & Creatividad \\
Mano derecha & \\
\hline
\end{tabular}

La postura corporal, la posición del instrumento tanto del violín como del arco, garantizará la práctica adecuada de todos los movimientos y el desarrollo del equilibrio natural para tocar. En cambio, un mal hábito en la postura acarreará problemas musculares y de tensión

(c) LUISA MOYA PASTOR. THE CONTENT OF THIS ARTICLE IS THE SOLE RESPONSIBILITY OF THE AUTHORS. THE REVISTA ELECTRÓNICA DE LEEME AND UNIVERSITAT DE VALĖNCIA ARE NOT LIABLE FOR ANY LEGAL ACTIONS THAT MAY ARISE INVOLVING THE ARTICLE'S CONTENT. REVISTA ELECTRÓNICA DE LEEME -LISTA ELECTRÓNICA EUROPEA DE MÚSICA EN LA EDUCACIÓN-HTTP://MUSICA.REDIRIS.ES.ISSN: 1575-9563 EDITORES: UNIVERSIDAD DE VALENCIA Y JESÚS TEJADA GIMÉNEZ. VISIBILIDAD DE ESTA REVISTA: EBSCO, CINDOC (CESIC), CITEFACTOR, COPAC, DIALNET, DICE (CSIC), DOAJ, E-REVISTAS (CSIC), EBSCO PREMIER, ERIH+, GALE CENGAGE LEARNING, IN-RECS, IRESIE, LATINDEX, MIAR, OCLC WORLDCAT, RESH, REDIB, RILM CORE JOURNALS, SUDOC, ULRICHS, ESTA REVISTA ESTA
INVESTIGACIONES CIENTIFICAS Y ES DE ACCESO LIBRE. CREATIVE COMMONS LICENSE 4.0 BY 


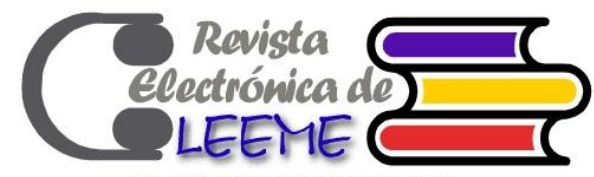

Electronic Journal of Music in Education

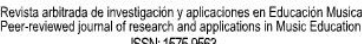

provenientes de este desequilibrio. Es necesario encontrar el equilibrio cuerpo-instrumento (Hoppenot, 2002).

Los movimientos de mano izquierda serán necesarios para una técnica básica de digitación. Los cambios de posición son los desplazamientos de la mano a lo largo del diapasón para tocar en otras posiciones. El alumnado debe familiarizarse con los armónicos (tonos producidos al tocar la cuerda al aire muy levemente en diversos puntos), que suelen anotarse con un pequeño círculo sobre la nota o en forma de diamante. Otro factor son los movimientos de mano derecha, es decir, los movimientos del arco: arco arriba y arco abajo; los golpes de arco, que son los diferentes ataques sobre la cuerda (martelé, spiccato, detaché, ligato, staccato); los cambios de cuerda que son los desplazamientos controlados del arco para cambiar de una cuerda a otra; y el control de la distribución del arco. Un recurso expresivo que se utiliza para embellecer el sonido es el vibrato (ondulación del sonido producida mediante un movimiento del dedo que pisa la cuerda e intensifica su vibración).

Respecto a los aspectos técnicos, la edad inicial para el aprendizaje del violín suele oscilar entre los 3-6 años, etapa decisiva porque su cerebro está más preparado para aprender nuevos procesos mentales y físicos. No pueden establecerse reglas muy rígidas porque cada sujeto es diferente y puede ser determinante la pasión, la fuerza de voluntad o la motivación, pero hay que desarrollar unas habilidades de coordinación y entrenar los músculos involucrados. De modo que, el tipo de clases, tanto individual como colectiva, es otro punto importante en estas metodologías. Tradicionalmente, la enseñanza se inicia bajo modalidad individual, ocupándose el profesorado de cada aspecto del aprendizaje técnico, repertorio adecuado, problemas de comprensión, asimilación, habilidad para mejorar sonoridad, afinación y musicalidad.

Según Evans (2015), la motivación está estrechamente relacionada con la autodeterminación y, para que ésta surja y se internalice, deben estar cubiertas las necesidades psicológicas básicas. Un espacio de comunicación abierto y liberal que dependerá del clima de seguridad, práctica del estímulo, desaparición del temor a crear delante de otros y la aceptación del análisis con atención a las propuestas del alumno, serán requisitos fundamentales. Para Martenot (1952), el gesto depende de la actitud interior y ésta del cuerpo, de un sistema nervioso y muscular que le permitirá exteriorizarse o no. Willems (1981) y Chevais (1932) han investigado sobre la importancia de la creatividad en los inicios musicales a partir de la práctica de la improvisación, al igual que Martenot (1993).

La lectura musical exige una atención y un esfuerzo mental especial y complejo. La mirada del músico debe prestar especial atención también a los signos que representan alturas, duraciones, unido a la rapidez de automatismos, compatibles con el desarrollo del movimiento sonoro, para poder expresar el sentido expresivo. La lectura musical está estrechamente vinculada a la memoria, ya que ésta se desarrolla desde el primer momento en el que se toca un

(c) LUISA MOYA PASTOR. THE CONTENT OF THIS ARTICLE IS THE SOLE RESPONSIBILITY OF THE AUTHORS. THE REVISTA ELECTRÓNICA DE LEEME AND UNIVERSITAT DE VALĖNCIA ARE NOT LABLE FOR ANY LEGAL ACTONS THAT MAY ARISE INVOLVING THE ARTICLE'S CONTENT. REVISTA ELECTRÓNICA DE LEEME -LISTA ELECTRÓNICA EUROPEA DE MÚSICA EN LA EDUCACIÓN-HTTP://MUSICA.REDIRIS.ES.ISSN: 1575-9563 EDITORES: UNIVERSIDAD DE VALENCIA Y JESÚS TEJADA GIMÉNEZ. VISIBILIDAD DE ESTA REVISTA: EBSCO, CINDOC (CESIC), CITEFACTOR, COPAC, DIALNET, DICE (CSIC), DOAJ, E-REVISTAS (CSIC), EBSCO PREMIER, ERIH+, GALE CENGAGE LEARNING, IN-RECS, IRESIE, LATINDEX, MIAR, OCLC WORLDCAT, RESH, REDIB, RILM CORE JOURNALS, SUDOC, ULRICHS, ESTA REVISTA ESTÁ PUBLICADA CON EL APOYO INSTITUCIONAL DE REDIRIS-CONSEJO SUPERIOR DE INVESTIGACIONES CIENTIFICAS Y ES DE ACCESO LIBRE. CREATIVE COMMONS LICENSE 4.0 BY 

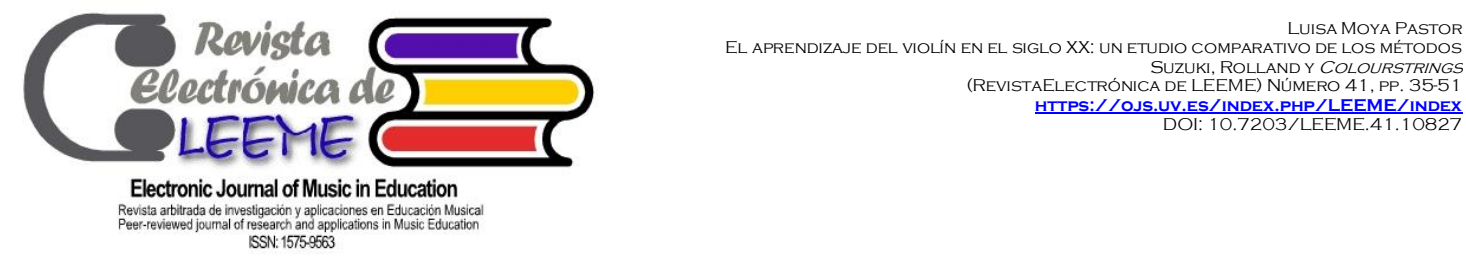

instrumento. Cuando el alumnado toca una pieza, no es suficiente con recordar las notas, las digitaciones o los golpes de arco, sino también las dinámicas, el fraseo y las sensaciones. La memorización es una experiencia global que comprende estrategias, estructuras y procesos que estarán fuertemente influenciadas por las características del instrumento y por los conocimientos del alumno.

En cuanto al repertorio, un factor de confianza reside en el éxito de los primeros ejercicios a realizar (Martenot, 1952): es mejor partir de un nivel inferior al alcanzado previamente, para luego pasar a mayor dificultad y evitar el fracaso inicial. Si los conceptos a transmitir son rígidos, se reflejará en la metodología de las clases y repercutirá en la creatividad. Así, otro aspecto es la creatividad. Los métodos de la pedagogía musical actual están basados en unos principios comunes: creatividad, motivación, componente lúdico, participación, imaginación, globalización. Koestler (1964) afirma que el acto creativo no surge de la nada: sería la interacción entre pensamiento y contexto sociocultural.

\subsection{Diseño de las entrevistas}

Para su diseño se han tenido en cuenta los aspectos agrupados en dos categorías (Tabla 1): un bloque de preguntas dedicado a los aspectos técnicos, para comprobar aplicación y desarrollo de recursos (postura corporal, afinación y sonido, ritmo, mano izquierda, cambios de posición y armónicos, vibrato y mano derecha); y otro bloque dedicado a los aspectos no técnicos, para profundizar en la experiencia docente y ampliar cuestiones relacionadas con el proceso de aprendizaje (edad inicial, tipo de clase, motivación, lectura musical y memoria, repertorio y creatividad). Estas entrevistas han sido útiles para contrastar los resultados entre metodologías, además de que han permitido profundizar en las prácticas docentes. De manera que, se han escogido dos docentes de cada metodología: profesionales Suzuki del Districte Marítim de Gandía y una profesora de la academia Cordes Espai Educatiu de Valencia; profesionales de Colourstrings del Conservatorio de Reus y la Escuela de Música Pau Casals del Vendrell. Del método Rolland no ha podido entrevistarse a nadie.

\section{Resultados}

\subsection{Aspectos técnicos}

Postura corporal: Suzuki y Rolland difieren en la postura corporal. Para Suzuki, la posición de descanso es distinta a la de tocar; en reposo los pies están juntos a la altura de los talones; al tocar, los talones están separados. Todos practican la misma posición (Figura 1) y mantienen el peso del instrumento en el costado izquierdo. En Rolland, es siempre la misma posición (Figura 2), buscando comodidad, flexibilidad y balanceo equitativo entre las dos partes

(C) LUISA MOYA PASTOR. THE CONTENT OF THIS ARTICLE IS THE SOLE RESPONSIBILITY OF THE AUTHORS. THE REVISTA ELECTRÓNICA DE LEEME AND UNIVERSITAT DE VALĖNCIA ARE NOT LIABLE FOR ANY LEGAL ACTIONS THAT MAY ARISE INVOLVING THE ARTICLE'S CONTENT. REVISTA ELECTRÓNICA DE LEEME -LISTA ELECTRÓNICA EUROPEA DE MÚSICA EN LA EDUCACIÓN-HTTP://MUSICA.REDIRIS.ES.ISSN: 1575-9563 EDITORES: UNIVERSIDAD DE VALENCIA Y JESÚS TEJADA GIMÉNEZ. VISIBILIDAD DE ESTA REVISTA: EBSCO, CINDOC (CESIC), CITEFACTOR, COPAC, DIALNET, DICE (CSIC), DOAJ, E-REVISTAS (CSIC), EBSCO PREMIER, ERIH+, GALE CENGAGE LEARNING, IN-RECS, IRESIE, LATINDEX, MIAR, OCLC WORLDCAT, RESH, REDIB, RILM CORE JOURNALS, SUDOC, ULRICHS, ESTA REVISTA ESTA
INVESTIGACIONES CIENTIFICAS Y ES DE ACCESO LIBRE. CREATIVE COMMONS LICENSE 4.0 BY 


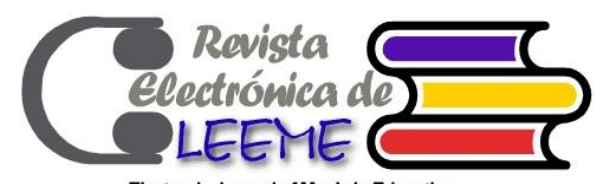

Electronic Journal of Music in Education

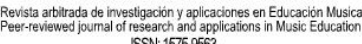

del cuerpo. Szilvay (2015) señala que una buena postura (Figura 3) requiere un equilibrio desde los pies hasta la cabeza, mientras que los pies deben estar ligeramente separados.

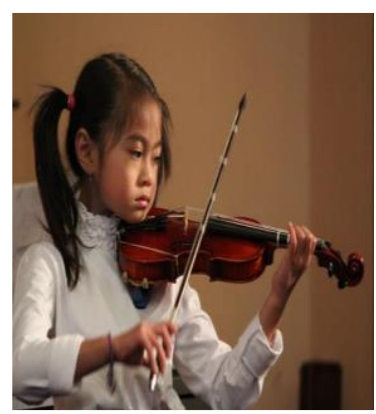

Figura 1. Postura Método Suzuki

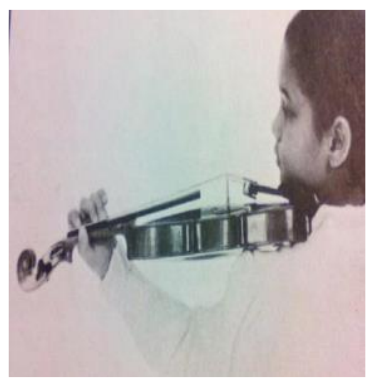

Figura 2. Postura Método Rolland (Johnson,1971, p.2)

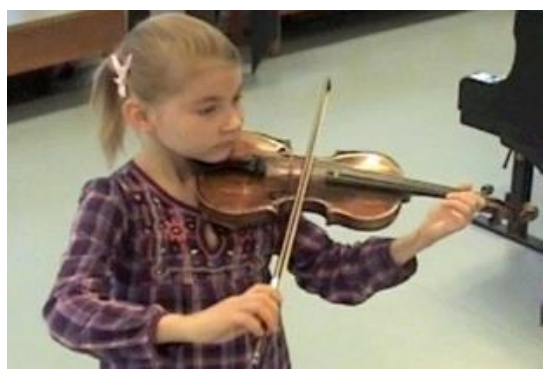

Figura 3. Postura Método Colourstrings

Afinación y sonido: Los conceptos de afinación y sonido se abordan de manera diferente en cada método. Suzuki (1969) se centra en la técnica del codo y en un sistema basado en marcas para controlar la afinación (Jansen Van Vuuren, 2016). En cuanto al desarrollo del sonido, se basa en la "tonalización", una serie de ejercicios de escalas, arpegios, cuerdas al aire y alguna melodía fácil que preparan al alumno para cada pieza. Además de esto, pone especial atención en el sonido, ya que a partir del repertorio desarrollará su musicalidad.

Rolland (1974) introduce las dificultades desde el principio, facilita que los movimientos sean libres, da importancia al equilibrio y al movimiento constante del cuerpo, al balanceo del peso, la cabeza, el brazo, el codo, las piernas, etc., para poder liberar tensión y producir buen sonido y una afinación correcta. La calidad del movimiento determina la calidad de sonido. Se colocan tres marcas en el diapasón para proporcionar al alumnado una orientación visual de la afinación sobre la línea del primer dedo, del tercero y en el centro del violín, en la posición del armónico (Johnson, 1971).

(c) LUISA MOYA PASTOR. THE CONTENT OF THIS ARTICLE IS THE SOLE RESPONSIBILITY OF THE AUTHORS. THE REVISTA ELECTRÓNICA DE LEEME AND UNIVERSITAT DE VALĖNCIA ARE NOT LIABLE FOR ANY LEGAL ACTIONS THAT MAY ARISE INVOLVING THE ARTICLE'S CONTENT. REVISTA ELECTRÓNICA DE LEEME -LISTA ELECTRÓNICA EUROPEA DE MÚSICA EN LA EDUCACIÓN-HTTP://MUSICA.REDIRIS.ES.ISSN: 1575-9563 EDITORES: UNIVERSIDAD DE VALENCIA Y JESÚS TEJADA GIMÉNEZ. VISIBILIDAD DE ESTA REVISTA: EBSCO, CINDOC (CESIC), CITEFACTOR, COPAC, DIALNET, DICE (CSIC), DOAJ, E-REVISTAS (CSIC), EBSCO PREMIER, ERIH+, GALE CENGAGE LEARNING, IN-RECS, IRESIE, LATINDEX, MIAR, OCLC
WORLDCAT, RESH, REDIB, RILM CORE JOURNALS, SUDOC, ULRICHS, ESTA REVISTA ESTÁ PUBLICADA CON EL APOYO INSTITUCIONAL DE REDIRIS-CONSEJO SUPERIOR DE INVESTIGACIONES CIENTIFICAS Y ES DE ACCESO LIBRE. CREATIVE COMMONS LICENSE 4.0 BY 

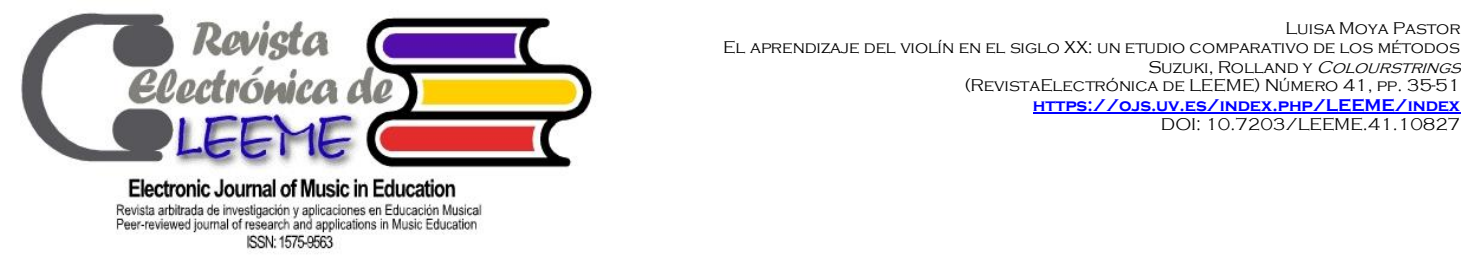

Colourstrings mantiene como principio el desarrollo del oído interno para una correcta afinación. A través de la "solmisación" se anima al niño a cantar primero lo que tocará después. Ello hace que relacione el sonido del instrumento con su propia voz y aprenda a escucharse (Voima, 2009). Desde el Book A se practica la transposición con el llamado "do móvil" para las tonalidades mayores y el "la móvil" para las tonalidades menores (Voima, 2009), desarrollándose el oído relativo.

Ritmo: En Suzuki, la formación rítmica se efectúa a través de ejercicios de imitación y repetición, recreándose y experimentándose así los patrones rítmicos. Mientras que, Rolland considera un factor muy importante la formación rítmica. Para desarrollar la expresión de la pulsación en las etapas iniciales, propone una serie de actividades para trabajar el aspecto rítmico como escoger una pieza con un ritmo definido, caminar con el ritmo de la pulsación haciendo movimientos amplios y libres, balancear los brazos y levantar los pies, hacer palmas llevando la pulsación, hacer lo mismo pero con acentos, gestos con la mano para dirigir la pulsación, practicar la marcha del estuche, hacer los golpes de arco simulados en un tubo o tocar en pizzicati con ambas manos y por último, tocarlo con el arco. Frente a esto, en Colourstrings, se utiliza la misma nomenclatura rítmica que en Kodály (Voima, 2009) y el concepto de ritmo se inicia a través de las imágenes conocidas. Mediante el empleo de grafías no convencionales el alumnado aprende de forma lúdica y amena (Figura 4).

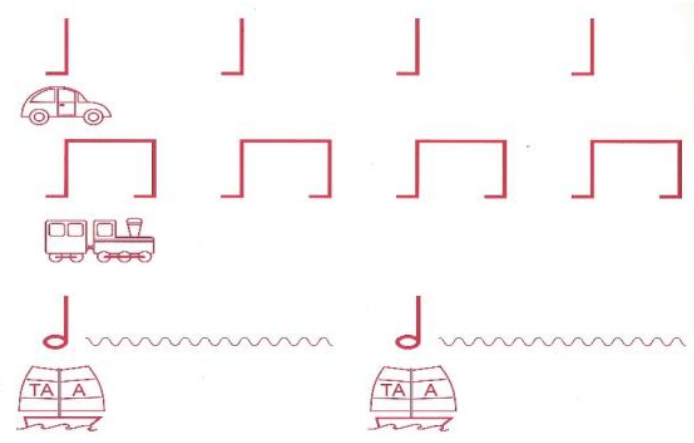

Figura 4. Representaciones rítmicas (Tomado de Szilvay, 2005, p.26)

Mano izquierda: La posición inicial de equilibrio para Rolland (1974) es con el segundo, tercer y cuarto dedo juntos, mientras que la de Suzuki y Colourstrings es con segundo y tercer dedo juntos. Pero, en Suzuki la posición es estática y el codo oscila para tocar en cada cuerda. Por su parte, Rolland (1974) diferencia tres tipos de movimientos de mano izquierda: el movimiento vertical (procura la puesta de los dedos), el movimiento horizontal (deslizamiento del mismo dedo) y el lateral (pasa un dedo de una cuerda a otra). Otro recurso pedagógico utilizado por Rolland es el pizzicato de mano izquierda, que ayuda a establecer una buena

(C) LUISA MOYA PASTOR. THE CONTENT OF THIS ARTICLE IS THE SOLE RESPONSIBILITY OF THE AUTHORS. THE REVISTA ELECTRÓNICA DE LEEME AND UNIVERSITAT DE VALĖNCIA ARE NOT LIABLE FOR ANY LEGAL ACTIONS THAT MAY ARISE INVOLVING THE ARTICLE'S CONTENT. REVISTA ELECTRÓNICA DE LEEME -LISTA ELECTRÓNICA EUROPEA DE MÚSICA EN LA EDUCACIÓN-HTTP://MUSICA.REDIRIS.ES.ISSN: 1575-9563 EDITORES: UNIVERSIDAD DE VALENCIA Y JESÚS TEJADA GIMÉNEZ. VISIBILIDAD DE ESTA REVISTA: EBSCO, CINDOC (CESIC), CITEFACTOR, COPAC, DIALNET, DICE (CSIC), DOAJ, E-REVISTAS (CSIC), EBSCO PREMIER, ERIH+, GALE CENGAGE LEARNING, IN-RECS, IRESIE, LATINDEX, MIAR, OCLC INVESTIGACIONES CIENTIFICAS Y ES DE ACCESO LIBRE. CREATIVE COMMONS LICENSE 4.0 BY 

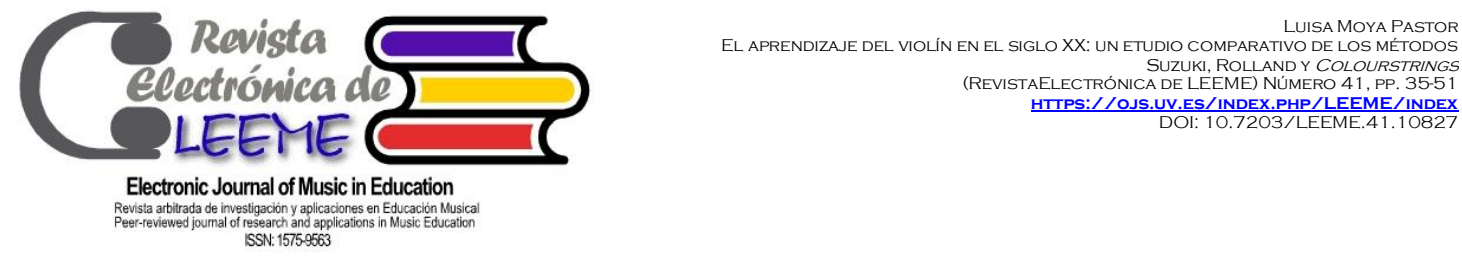

posición de ésta y a desarrollar la fuerza necesaria, flexibilidad e independencia digital, especialmente del cuarto dedo.

En Colourstrings, se utilizan pizzicati de mano izquierda para aprender la técnica básica desde el Book A (Figura 5); esto hace que los dedos adquieran la fuerza necesaria para pisar la cuerda progresivamente (Szilvay, 2005). Según Vooima (2009) el método da gran importancia al desarrollo del cuarto dedo a través del uso de los pizzicati.

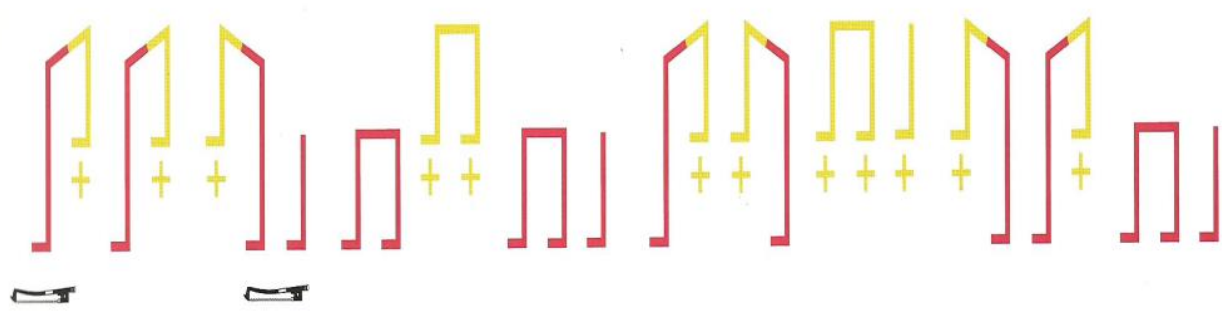

Figura 5. Uso de los pizzicati combinado con el arco (Szilvay, 2005, p.42)

Cambios de posición y armónicos: La técnica de los cambios de posición es otra diferencia significativa. En Suzuki, se introducen en el Volumen 4 (Starr, 1976) y se comienza con la tercera posición, pero en los Volúmenes 2 y 3 ya se desplaza la mano para realizar los armónicos naturales. Ya en Rolland, se enseña a tener una movilidad de la mano desde los comienzos para no anclarse en una posición determinada, estableciéndose tres posiciones desde las primeras clases: baja, media y alta. Mientras en Colourstrings, se utilizan los armónicos naturales (Figura 6) del violín desde el Book A y se incorporan algunas de las ideas de Rolland. Por ejemplo, se busca mediante la práctica de estos armónicos, habilidad y flexibilidad en el sistema básico de la mano izquierda. Mediante estos ejercicios, el alumnado se familiariza con las diferentes posiciones del diapasón, evitando que se aferre al mango del violín, problema común entre los principiantes.

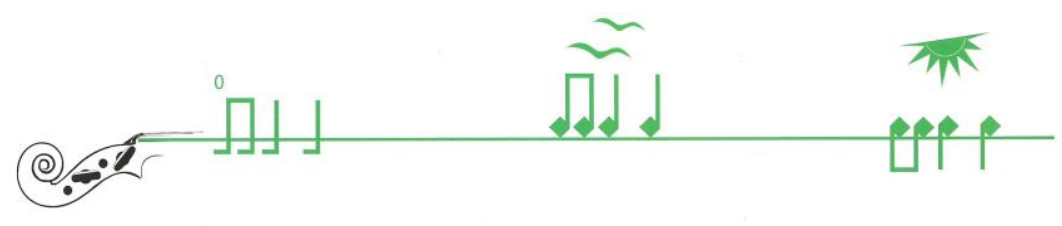

Figura 6. Representación de los armónicos naturales (Szilvay, 2005, p.34) 


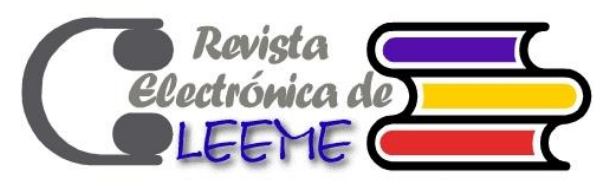

Electronic Journal of Music in Education

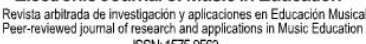

Vibrato: Respecto al vibrato, en Suzuki aparece en el Volumen 4, en el mismo momento en que se aprenden los cambios de posición. En Rolland, el vibrato se inicia como herramienta para eliminar tensiones de la mano y debe ser regular, y la velocidad y la amplitud pueden variar, originándose el vibrato estrecho, medio o ancho. En Colourstrings, aparece de manera asistida desde el primer año para liberar tensiones en el brazo.

Mano derecha: La posición de la mano derecha para Suzuki y en Colourstrings es la misma para los principiantes: el pulgar sujeta el arco por debajo de las crines (Figura 7); mientras que, en Rolland, se sitúa en el punto de equilibrio (Figura 8): introduce los golpes de arco y el concepto de pizzicato volante.

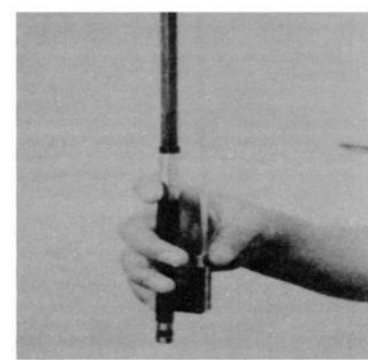

Figura 7. Posición del arco en Suzuki y en Colourstrings (Suzuki, 1959, p.4)

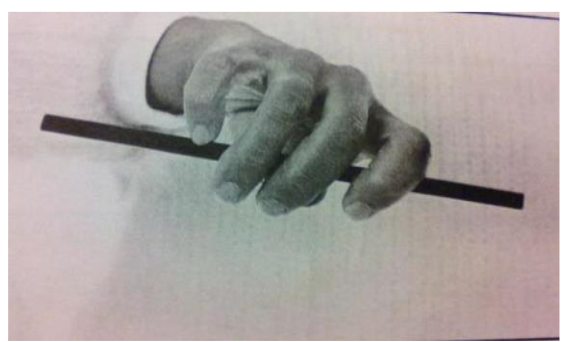

Figura 8. Posición del arco en Rolland (Johnson, 1971, p.7)

Tabla 2. Comparativa de aspectos técnicos en la iniciación del violín

\begin{tabular}{|c|c|c|c|}
\hline & Suzuki & Rolland & Colourstrings \\
\hline Postura corporal & $\begin{array}{l}\text { En reposo: pies en "V" y } \\
\text { violín inclinado en } \\
\text { reposo. Al tocar talones } \\
\text { separados. El peso en la } \\
\text { parte izquierda. }\end{array}$ & $\begin{array}{l}\text { En reposo: voluta no muy } \\
\text { baja. Al tocar: equilibrada y } \\
\text { busca el movimiento con } \\
\text { transferencia del peso para } \\
\text { liberar tensiones. }\end{array}$ & $\begin{array}{l}\text { Equilibrio desde los } \\
\text { pies hasta la cabeza, } \\
\text { pies deben estar } \\
\text { ligeramente separados. }\end{array}$ \\
\hline $\begin{array}{l}\text { Afinación } \\
\text { y sonido }\end{array}$ & $\begin{array}{l}\text { Afinación: uso de } \\
\text { pegatinas para su control. } \\
\text { Sonido: imi-tación, } \\
\text { "tonalización"y otros } \\
\text { ejercicios. }\end{array}$ & $\begin{array}{l}\text { Afinación: usa tres pegatinas } \\
\text { como guía. Sonido: calidad } \\
\text { del sonido a través de } \\
\text { relajación y compensación } \\
\text { de movimientos. }\end{array}$ & $\begin{array}{l}\text { Afinación:desarrollo } \\
\text { del oído interno a } \\
\text { partir de la“solmi- } \\
\text { sación”. Sonido de } \\
\text { gran calidad. }\end{array}$ \\
\hline Mano Izquierda & $\begin{array}{l}\text { Posición estática, con el } \\
\text { codo que oscila para cada } \\
\text { cuerda. Equilibrio de la } \\
\text { mano entre los dedos } 2^{\circ} \text { y } \\
3^{\circ} \text {. }\end{array}$ & $\begin{array}{l}\text { Equilibrio de la mano entre } \\
\text { los dedos } 2^{\circ}, 3^{\circ} \text { y } 4^{\circ} \text {. } \\
\text { Distingue tres tipos de } \\
\text { movimientos de dedo. } \\
\text { Utiliza pizzicati de mano } \\
\text { izquierda. }\end{array}$ & $\begin{array}{l}\text { Equilibrio de la mano } \\
\text { entre los dedos } 2^{\circ} \text { y } 3^{\circ} \text {. } \\
\text { Importancia del } \\
\text { desarrollo del } 4^{\circ} \text { dedo } \\
\text { en pizzicati. }\end{array}$ \\
\hline
\end{tabular}

(C) LUISA MOYA PASTOR. THE CONTENT OF THIS ARTICLE IS THE SOLE RESPONSIBILITY OF THE AUTHORS. THE REVISTA ELECTRÓNICA DE LEEME AND UNIVERSITAT DE VALĖNCIA ARE NOT LIABLE FOR ANY LEGAL ACTIONS THAT MAY ARISE INVOLVING THE ARTICLE'S CONTENT. REVISTA ELECTRÓNICA DE LEEME -LISTA ELECTRÓNICA EUROPEA DE MÚSICA EN LA EDUCACIÓN-HTTP://MUSICA.REDIRIS.ES.ISSN: 1575-9563 EDITORES: UNIVERSIDAD DE VALENCIA Y JESÚS TEJADA GIMÉNEZ. VISIBILIDAD DE ESTA REVISTA: EBSCO, CINDOC (CESIC), CITEFACTOR, COPAC, DIALNET, DICE (CSIC), DOAJ, E-REVISTAS (CSIC), EBSCO PREMIER, ERIH+, GALE CENGAGE LEARNING, IN-RECS, IRESIE, LATINDEX, MIAR, OCLC WORLDCAT, RESH, REDIB, RILM CORE JOURNALS, SUDOC, ULRICHS, ESTA REVISTA ESTÁ PUBLICADA CON EL APOYO INSTITUCIONAL DE REDIRIS-CONSEJO SUPERIOR DE INVESTIGACIONES CIENTIFICAS Y ES DE ACCESO LIBRE. CREATIVE COMMONS LICENSE 4.0 BY 

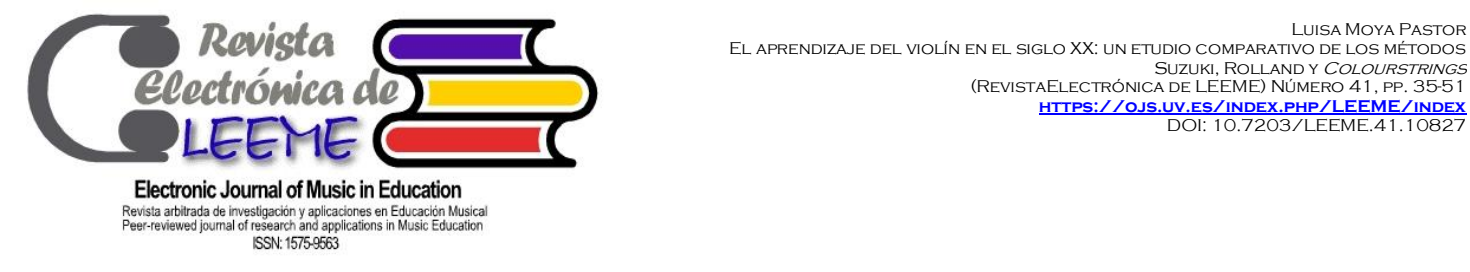

Vibrato

A la vez que los cambios de posición.

\section{Cambios \\ de posición \\ y armónicos}

Mano derecha

Ritmo

\begin{abstract}
Al consolidar la técnica básica de primera posición en el volumen 4. Los armónicos naturales aparecen desde el volumen 2.

Posición del debutante distinta a la del avanzado.

Sí, patrones rítmicos de imitación y repetición.
\end{abstract}

Como herramienta para eliminar tensiones de la mano, debe ser regular, la velocidad y la amplitud pueden variar.

\section{Sí, para facilitar la} movilidad de la mano. Tres posiciones desde los comienzos: baja, media y alta.

Desde el principio de manera asistida liberando el brazo de tensiones.

\author{
Sí, los armónicos \\ naturales aparecen \\ desde el book $A$. \\ Paso del arco de \\ manera asistida por el \\ profesor. \\ Sí, con representación \\ visual y grafías no \\ convencionales.
}

Iniciación en los golpes de arco y en el pizzicato volante.

Sí, con juegos rítmicos y una acción rítmica precisa.

\subsection{Aspectos no técnicos}

Edad inicial: Como se ha señalado, suele fluctuar entre los 3-6 años. Al comparar este aspecto en los métodos, se ha observado que el que comienza más temprano es Suzuki, situándose entre los 3-4 años. Esto no es de extrañar pues una parte importante de su enfoque musical se basa en crear un entorno musical donde se aprenda la música como la "lengua materna”. Ante esto, Rolland parte de los 6 años porque su método está centrado en el desarrollo del cuerpo y la conciencia del movimiento: la edad no es tan importante, incluso se especializó en lo que él denominaba "reciclajes" de alumnado de edad avanzada (14 -15 años) ayudándoles a mejorar su técnica. Y, en Colourstrings, comienzan a partir de los 5 años: ña relevancia de este aspecto es el desarrollo de los sentidos musicales a través de un ambiente agradable y cálido, donde el alumnado responde a los estímulos musicales desde la naturalidad.

Tipo de clase: En Suzuki, se incluyen semanalmente dos clases instrumentales (individual y colectiva) de 20 minutos cada una. La presencia de los padres es fundamental e, incluso, reciben clase para poder ayudar y guiar el estudio. Suzuki y Rolland coinciden en que la clase colectiva está estrechamente vinculada a la motivación para aprender del compañero a través de la observación, ejercita el oído, repasa el material aprendido y domina el repertorio; sin embargo, el profesorado entrevistado coincide que estas sesiones no pueden sustituir a las individuales. Hay muchos aspectos y diferencias que no se pueden aprender en una clase grupal (sonido o afinación, adaptación al ritmo del aprendizaje).

Rolland fue uno de los primeros pedagogos en destacar la importancia del aprendizaje en grupo y le daba tanta importancia como al individual; y, en su método, no se habla del tiempo de duración de las clases, pero señala que deben ser dos clases semanales (individual y colectiva). En Colourstrings se empieza con la enseñanza individual y, luego, la completa con la

(c) LUISA MOYA PASTOR. THE CONTENT OF THIS ARTICLE IS THE SOLE RESPONSIBILITY OF THE AUTHORS. THE REVISTA ELECTRÓNICA DE LEEME AND UNIVERSITAT DE VALĖNCIA ARE NOT LIABLE FOR ANY LEGAL ACTIONS THAT MAY ARISE INVOLVING THE ARTICLE'S CONTENT. REVISTA ELECTRÓNICA DE LEEME -LISTA ELECTRÓNICA EUROPEA DE MÚSICA EN LA EDUCACIÓN-HTTP://MUSICA.REDIRIS.ES.ISSN: $1575-9563$ EDITORES: UNIVERSIDAD DE VALENCIA Y JESÚS TEJADA GIMÉNEZ. VISIBILIDAD DE ESTA REVISTA: EBSCO, CINDOC (CESIC), CITEFACTOR, COPAC, DIALNET, DICE (CSIC), DOAJ, E-REVISTAS (CSIC), EBSCO PREMIER, ERIH+, GALE CENGAGE LEARNING, IN-RECS, IRESIE, LATINDEX, MIAR, OCLC INVESTIGACIONES CIENTIFICAS Y ES DE ACCESO LIBRE. CREATIVE COMMONS LICENSE 4.0 BY 

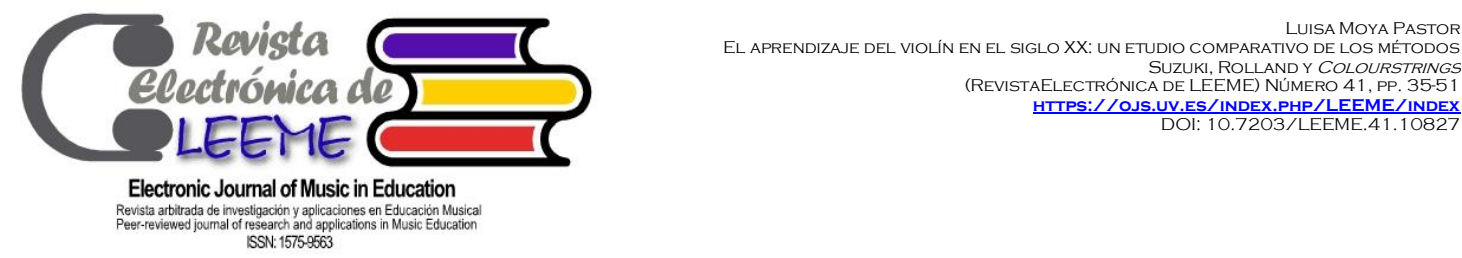

de grupo, para introducir al niño en la ejecución de música de cámara. Las clases son de 45 minutos cada una (individual y colectiva con 5-9 estudiantes), abiertas a los padres.

Motivación: Según Suzuki (1969), los niños aprenden reaccionando a los estímulos de su ambiente y a través de los sentidos, no de forma intelectual: el ambiente es lo que motiva y la motivación facilitará el aprendizaje que se sustenta a través del triángulo de aprendizaje: padreprofesor-alumno. En Rolland, la motivación aparece vinculada a las clases colectivas y al repertorio. Y, Szilvay (1996) sostiene que el método Colourstrings crea un entorno donde juguetes, cuentos, canciones e instrumentos están al servicio de la felicidad del niño. Según Vooima (2009) la música es ofrecida como un "paquete artístico", en el que el desarrollo de la técnica musical y las emociones se producen todo el tiempo de un modo equilibrado, empleando explicaciones visuales para las nociones más complejas.

Lectura musical y memoria: Suzuki y Rolland coinciden en que la lectura y la memoria son imprescindibles. Suzuki aborda la lectura en el Volumen 4 cuando el niño tiene desarrollada su habilidad para escuchar y reproducir sonidos, tocando un repertorio de memoria $\mathrm{y}$ le introduce en la lectura del repertorio aprendido, relacionando frases y elementos y estableciendo relaciones visuales, auditivas, rítmicas y armónicas y también la lectura a primera vista. En cambio, Rolland la introduce desde el principio, apoyándose en la formación rítmica a través del movimiento corporal. Mientras que, en Colourstrings, la lectura es esencial y aparece simplificada con colores e imágenes; primeramente, se trabaja el aspecto rítmico y gradualmente se añaden las líneas del pentagrama. Voima (2009) señala cómo Szilvay introduce los contenidos visualmente, a través de imágenes e ilustraciones (Figura 9) y conecta lectoescritura musical con técnica instrumental mediante el juego. La coordinación motora se desarrolla a medida que avanzan los procesos intelectuales. No se potencia la memorización en las fases iniciales, sino que los niños desarrollan sus habilidades memorísticas a través del aprendizaje memorístico de canciones, de la relación de ritmos con imágenes o del conocimiento del instrumento sin el control visual.
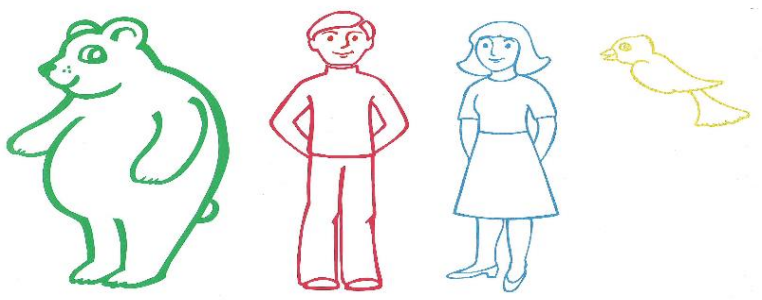

Figura 9. Correspondencia visual con las cuerdas: SOL-RE-LA-MI· (Szilvay, 2005, p.1) 


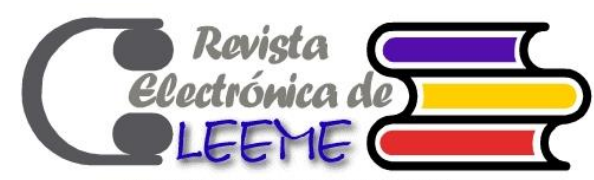

Electronic Journal of Music in Education

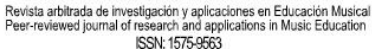

Repertorio: En Suzuki, se utilizan piezas clásicas y barrocas durante los diez volúmenes, que guían el aprendizaje de forma secuenciada. El repertorio en los primeros libros se aprende por imitación; y el alumnado comienza memorizando piezas cortas y a medida que progresa, sigue reteniendo y memorizando las anteriores, con lo que ejercita constantemente la memoria y la concentración, lo que hace que la interpretación del instrumento sea más fluida.

El material de Rolland resulta atractivo y divertido para el alumnado, con canciones populares y música contemporánea del compositor y arreglista Fletcher; aunque no está secuenciado progresivamente, es ideal para trabajar aspectos técnicos concretos: se distribuye la información en diferentes bloques o acciones dedicados a la posición, vibrato, cambios de posición, golpes de arco, etc.; y cada bloque trata un tema diferente y el profesorado organiza el material utilizando el "currículum espiral". Mientras que, en Colourstrings, el material didáctico está muy bien secuenciado. Los primeros pasos comienzan con la sensibilización musical en el jardín de infancia: utiliza los "little Rascals"; seguidamente, utiliza canciones populares alternando con clásicas y barrocas e, incluso, contemporáneas (Rossa, discípulo de Kodály).

Creatividad: En el método Suzuki y el método Rolland, no se contempla la creatividad como tal. Mientras que, en Colourstrings, la creatividad se desarrolla por medio de la transposición, improvisación y composición.

Seguidamente, la Tabla 3 muestra una comparativa sintetizada de las tres metodologías investigadas a lo largo de este documento:

Tabla 3. Comparativa de aspectos no técnicos en la iniciación del violín

\begin{tabular}{|c|c|c|c|}
\hline & Suzuki & Rolland & Colourstrings \\
\hline Edad inicial & 3-4 años & 6 años & 5 años \\
\hline Tipo de clases & $\begin{array}{l}\text { Individual y colectiva, de } 20 \\
\text { minutos cada una. }\end{array}$ & $\begin{array}{l}\text { Individual y colectiva, no } \\
\text { figura el tiempo. }\end{array}$ & $\begin{array}{l}\text { Individual y colectiva, de } 45 \\
\text { minutos cada clase. }\end{array}$ \\
\hline Motivación & $\begin{array}{l}\text { Sí, por parte del profesor y } \\
\text { padres. Importancia del } \\
\text { ambiente y triángulo de } \\
\text { aprendizaje. }\end{array}$ & $\begin{array}{l}\text { A través de las clases } \\
\text { colectivas se mantiene } \\
\text { motivados a los alumnos. }\end{array}$ & $\begin{array}{l}\text { Sí. La música se ofrece al } \\
\text { niño como "paquete } \\
\text { artístico". Las clases están } \\
\text { abiertas a los padres. }\end{array}$ \\
\hline $\begin{array}{l}\text { Lectura musical } \\
\text { y memoria }\end{array}$ & $\begin{array}{c}\text { Aprendizaje por imitación al } \\
\text { principio. Aborda la lectura } \\
\text { en el volumen } 4 .\end{array}$ & $\begin{array}{l}\text { Imprescindible, comienza la } \\
\text { lectura combinada con la } \\
\text { formación rítmica. }\end{array}$ & $\begin{array}{c}\text { Sí, con colores de las cuerdas } \\
\text { y la representación de grafías } \\
\text { no convencionales. }\end{array}$ \\
\hline Repertorio & $\begin{array}{c}\text { Aprendizaje progresivo en } \\
10 \text { volúmenes. Piezas } \\
\text { barrocas y clásicas }\end{array}$ & $\begin{array}{l}\text { Técnica condensada en dos } \\
\text { años. Canciones populares y } \\
\text { música contemporánea. }\end{array}$ & $\begin{array}{c}\text { Books por niveles (A-F), } \\
\text { dúos y música de cámara. } \\
\text { Canciones populares, piezas } \\
\text { barrocas y clásicas. }\end{array}$ \\
\hline Creatividad & No lo contempla & No lo contempla & $\begin{array}{l}\text { Sí. Transposición, } \\
\text { improvisación y } \\
\text { composición. } \\
\end{array}$ \\
\hline
\end{tabular}

(C) LUISA MOYA PASTOR. THE CONTENT OF THIS ARTICLE IS THE SOLE RESPONSIBILITY OF THE AUTHORS. THE REVISTA ELECTRÓNICA DE LEEME AND UNIVERSITAT DE VALĖNCIA ARE NOT LIABLE FOR ANY LEGAL ACTIONS THAT MAY ARISE INVOLVING THE ARTICLE'S CONTENT. REVISTA ELECTRÓNICA DE LEEME -LISTA ELECTRÓNICA EUROPEA DE MÚSICA EN LA EDUCACIÓN-HTTP://MUSICA.REDIRIS.ES.ISSN: 1575-9563 EDITORES: UNIVERSIDAD DE VALENCIA Y JESÚS TEJADA GIMÉNEZ. VISIBILIDAD DE ESTA REVISTA: EBSCO, CINDOC (CESIC), CITEFACTOR, COPAC, DIALNET, DICE (CSIC), DOAJ, E-REVISTAS (CSIC), EBSCO PREMIER, ERIH+, GALE CENGAGE LEARNING, IN-RECS, IRESIE, LATINDEX, MIAR, OCLC INVESTIGACIONES CIENTIFICAS Y ES DE ACCESO LIBRE. CREATIVE COMMONS LICENSE 4.0 BY 

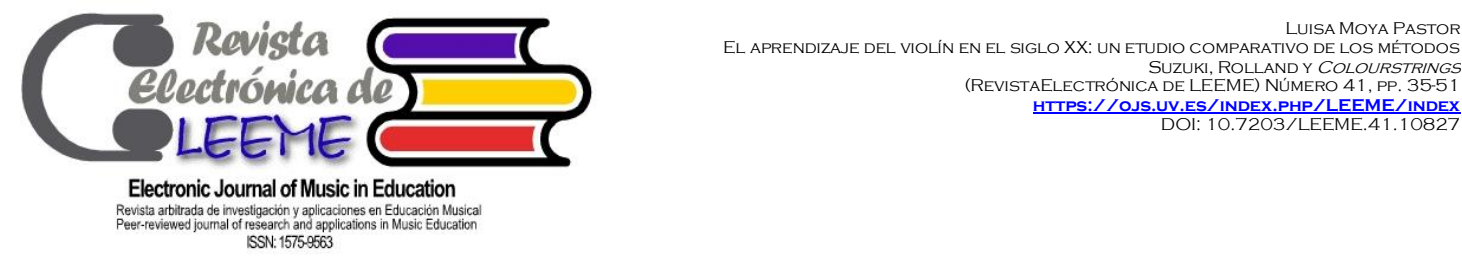

\section{Discusión}

Todos estos métodos persiguen el mismo objetivo: el aprendizaje del violín, pero de diferente forma. Esto ha llevado a ciertas reflexiones:

1. Cuanto más pronto se comienza, más necesario se hace secuenciar los contenidos. En este sentido, resulta acertada la manera de replantearlos en Colourstrings, de forma que resulten inteligibles para el niño.

2. La utilización de las clases colectivas constituye un gran pilar de motivación, siempre complementado con clases individuales donde tengan su espacio de aprendizaje personalizado.

3. La utilización de los cambios de posición y de los armónicos de Colourstrings es un aspecto relevante, porque en Enseñanzas Elementales no suele abordarse hasta tercer curso.

4. La lectura musical y su relación con la transposición, o con la creatividad, hace que el alumnado aprenda y viva los contenidos, disfrutando de la música e iniciándose en el proceso creativo.

5. Contar con un amplio repertorio aporta múltiples posibilidades. Es relevante la propuesta de Jansen van Vuuren (2016) de práctica combinada de lectura musical del Volumen 1 de Suzuki con el Book B Colourstrings.

En suma, para elaborar una propuesta pedagógica se aunaría el desarrollo rítmico, el vibrato y el movimiento de Rolland, sumados con el desarrollo de la memoria y la motivación de Suzuki, con los principios de lectura, repertorio, creatividad, cambios de posición y armónicos de Colourstrings. Además de esto, las clases se estructurarían en dos clases semanales, de 30 minutos individual y de 30 minutos grupal con un máximo de 8 estudiantes con una edad inicial de 4-5 años.

\section{Conclusiones}

Se han elegido estas tres metodologías por considerar que son las más apropiadas para la iniciación al violín. Actualmente, en España, las pedagogías Suzuki y Rolland están siendo aplicadas en numerosas escuelas de música y conservatorios, pero el uso del método Coloustrings es minoritario. Gracias al análisis comparativo se han resaltado ciertos ítems fundamentales en dicho proceso y se han conectado ideas, lo cual ha servido para establecer una propuesta pedagógica.

(c) LUISA MOYA PASTOR. THE CONTENT OF THIS ARTICLE IS THE SOLE RESPONSIBILITY OF THE AUTHORS. THE REVISTA ELECTRÓNICA DE LEEME AND UNIVERSITAT DE VALĖNCIA ARE NOT LIABLE FOR ANY LEGAL ACTIONS THAT MAY ARISE INVOLVING THE ARTICLE'S CONTENT. REVISTA ELECTRÓNICA DE LEEME -LISTA ELECTRÓNICA EUROPEA DE MÚSICA EN LA EDUCACIÓN-HTTP://MUSICA.REDIRIS.ES.ISSN: 1575-9563 EDITORES: UNIVERSIDAD DE VALENCIA Y JESÚS TEJADA GIMÉNEZ. VISIBILIDAD DE ESTA REVISTA: EBSCO, CINDOC (CESIC), CITEFACTOR, COPAC, DIALNET, DICE (CSIC), DOAJ, E-REVISTAS (CSIC), EBSCO PREMIER, ERIH+, GALE CENGAGE LEARNING, IN-RECS, IRESIE, LATINDEX, MIAR, OCLC WORLDCAT, RESH, REDIB, RILM CORE JOURNALS, SUDOC, ULRICHS, ESTA REVISTA ESTÁ PUBLICADA CON EL APOYO INSTITUCIONAL DE REDIRIS-CONSEJO SUPERIOR DE INVESTIGACIONES CIENTIFICAS Y ES DE ACCESO LIBRE. CREATIVE COMMONS LICENSE 4.0 BY 

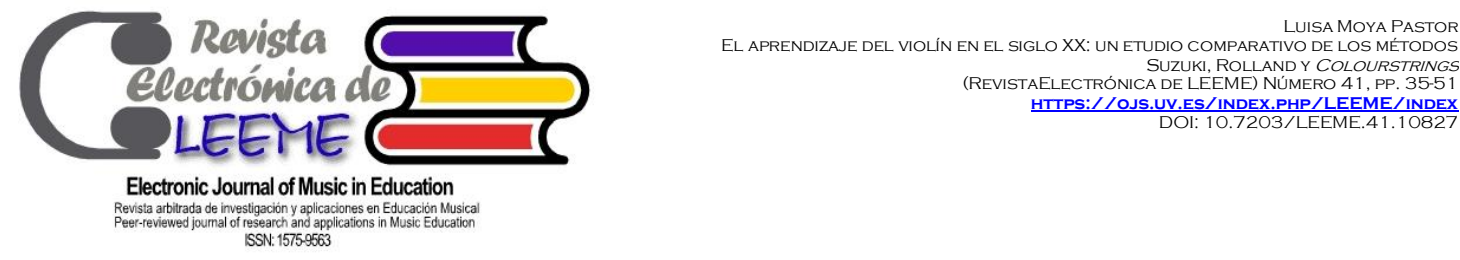

Si se observan sus principios generales, pueden verse muchas diferencias, pero si se ahonda se contempla cómo también convergen e, incluso, pueden complementarse para crear otras propuestas pedagógicas, en aras de la personalización. Por ejemplo, la visión integral del aprendizaje como un trabajo educativo a todos los niveles es muy relevante en Suzuki y Colourstrings; y, este planteamiento es más completo globalmente que el de Rolland, focalizado en el aprendizaje técnico específico durante los dos primeros años. Otro aspecto relevante en Suzuki es que se respeta el ritmo individual de aprendizaje, realizando conciertos con objetivos integradores, en los que tocan niños de todos los niveles y pequeños con mayores; en Colourstrings, en cambio, se motiva al alumnado siendo exigentes y valorando cada logro. Por otro lado, tras el análisis, haciendo referencia a los aspectos no técnicos, los objetivos de Suzuki y Colourstrings están más claramente definidos y motivan más que en Rolland. Otra observación tras el análisis es lo apropiado de la propuesta de Rolland y Colourstrings de introducir ciertas dificultades técnicas casi desde el comienzo (armónicos, cambios de posición y vibrato), que favorecen la relajación de la mano izquierda.

La realización de las entrevistas ha permitido profundizar sobre la práctica de estas metodologías. En Suzuki y Colourstrings, el profesorado entrevistado otorga una gran importancia a su formación por especialistas: en la práctica del aula, enseñan a dar clases a los más jóvenes, se observan y se cuestionan constantemente, para hacer que la enseñanza evolucione. El amplio material pedagógico de Colourstrings es otro aspecto que han destacado, por facilitar el desarrollo de las clases y constituir una guía pedagógica que contempla todos los aspectos del aprendizaje. Y, con respecto a la introducción de la lectura musical, los profesionales Suzuki comentan que la lectura musical debe introducirse cuando se considere que el alumnado está preparado; sin embargo, la lectura en Colourstrings a partir de notación no convencional, conecta la parte emocional con la intelectual y resulta un factor primordial para el aprendizaje porque aprende a través de su experiencia. Esto hace que su creatividad crezca y aprenda jugando con especial motivación.

El aprendizaje de un instrumento no tiene barreras, ni dogmas o escuelas rígidas e inamovibles, sino que está en constante evolución y cuestionamiento. Cada docente, con sus vivencias, experiencias y conocimientos, es el principal responsable de encontrar y distribuir las herramientas pedagógicas para contribuir a la formación del alumnado. Se hace indispensable que el profesorado sea flexible, pues desde los inicios del aprendizaje habrá de contagiar la pasión por la música, y ayudar a consolidar cimientos técnicos y no técnicos que acompañarán al violinista a lo largo de toda su vida.

\section{Referencias}

Chevais, M. (1932). La nouvelle Education. París: Leduc. 

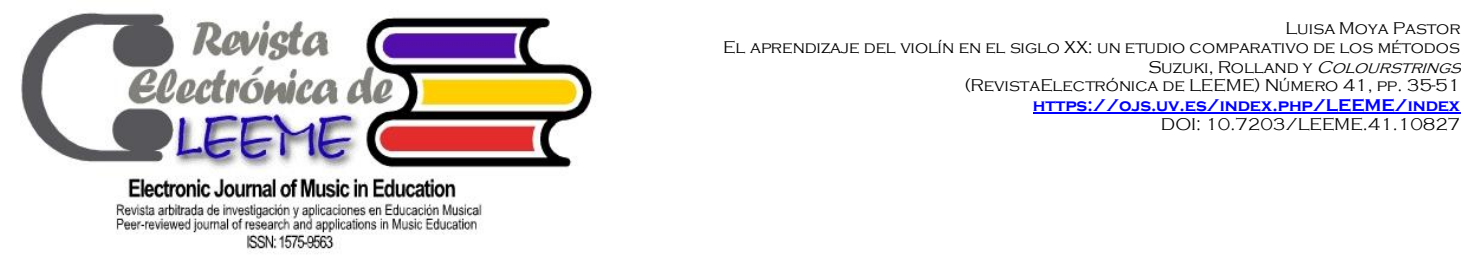

Evans, P. (2015). Self-determination theory: An approach to motivation in music education. Musicae Scientiae, 19 (1), 65-83.

Fanelli, M. (2011). Paul Rolland 1911-2011: A century Celebration. American String Teacher, 61 (1), 20-23.

Fray, D. L. (1979). An annotated bibliography for string teachers: 100 selected sources from string pedagogy, physiology, and related disciplines (1929-1978). Rochester, NY: University of Rochester, Eastman School of Music.

Göktürk, C. D. (2011). Comparing two contemporary violin teaching methods: Suzuki and Rolland. Kastamonu Education Journal, 19 (2), 401-408.

Haun, S. C. (1988). An annotated bibliography for violin teachers. University of Miami: Miami.

Hoppenot, D. (2002). El violín interior. Madrid: Real Musical.

Jansen van Vuuren, C. (2016). A structured comparison between the Suzuki and Colourstrings violin methods with critical reference to the teaching of notation reading skills/ Two examination concerts. Pretoria: Universidad de Pretoria.

Johnson, S. (1971). Young Strings in Action Paul's Rolland Approach to String Playing. New York: Boosey and Hawkes.

Kodály, F. (1964). The Selected Writings of Zoltán Kodály. New York: Boosey \& Hawkes.

Koestler, A. (1964). The Art of Creation. London: Hutchison and CO.

Kuo, Y. y Chuang, M. (2013). A proposal of a colour music notation system on a single melody for music beginners. International Journal of Music Education, 31 (4), 394-412.

Martenot, M. (1952). Formation et développement musical solfège: méthode Martenot. París: Magnard.

Martenot, M. (1993). Principios fundamentales de formación musical y su aplicación. Madrid: Rialp.

Mitchell, B. S. (1994). A qualitative study of Geza Szilvay's colourstrings method for violin. (Tesis doctoral). Recuperada de ProQuest Dissertations (304121956).

Nelson, J. (2011). An Investigation of Mimi Zweig's Violin Pedagogy and its Application to the American Public School String Orchestra Classroom in the 21st Century. Florida: University of Florida.

(C) LUISA MOYA PASTOR. THE CONTENT OF THIS ARTICLE IS THE SOLE RESPONSIBILITY OF THE AUTHORS. THE REVISTA ELECTRÓNICA DE LEEME AND UNIVERSITAT DE VALĖNCIA ARE NOT LIABLE FOR ANY LEGAL ACTIONS THAT MAY ARISE INVOLVING THE ARTICLE'S CONTENT. REVISTA ELECTRÓNICA DE LEEME -LISTA ELECTRÓNICA EUROPEA DE MÚSICA EN LA EDUCACIÓN-HTTP://MUSICA.REDIRIS.ES.ISSN: 1575-9563 EDITORES: UNIVERSIDAD DE VALENCIA Y JESÚS TEJADA GIMÉNEZ. VISIBILIDAD DE ESTA REVISTA: EBSCO, CINDOC (CESIC), CITEFACTOR, COPAC, DIALNET, DICE (CSIC), DOAJ, E-REVISTAS (CSIC), EBSCO PREMIER, ERIHH, GALE CENGAGE LEARNING, IN-RECS, IRESIE, LATINDEX, MIAR, OCLC WORLDCAT, RESH, REDIB, RILM CORE JOURNALS, SUDOC, ULRICHS, ESTA REVISTA ESTÁ PUBLICADA CON EL APOYO INSTITUCIONAL DE REDIRIS-CONSEJO SUPERIOR DE INVESTIGACIONES CIENTIFICAS Y ES DE ACCESO LIBRE. CREATIVE COMMONS LICENSE 4.0 BY 

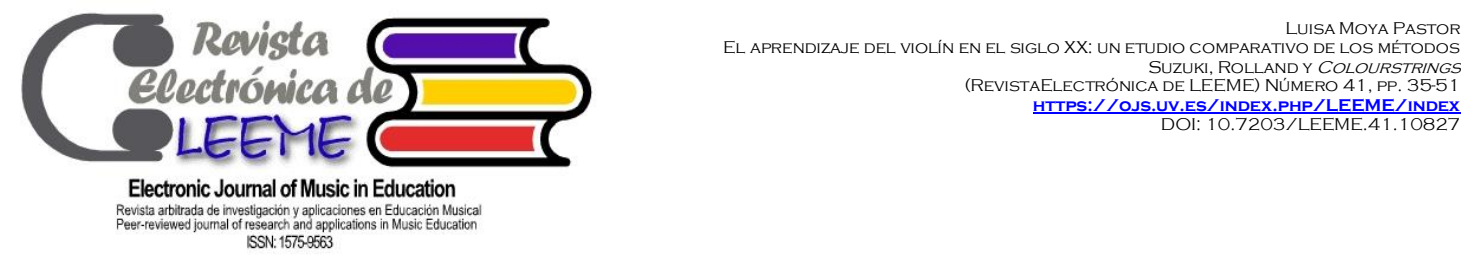

Perkins, M. M. (1995). A comparison of violin playing techniques: Kato Havas, Paul Rolland and Shinichi Suzuki. Bloomington, Indiana: American String Teachers Association.

Rolland, P. (1959). Basics Principles of Violin Playing. Washington: Music Educators National Conference.

Rolland, P., Krolick, E., y Rowell, M. (1971). Prelude to string playing. New York: Boosey \& Hawkes.

Rolland, P. y Mutschler, M. (1974). The teaching of Action in String Playing: Developmental and remedial techniques. Urbana, Illinois: Illinois String Research Associates.

Ruokonen, I., Juntunen, P. y Ruismäki, H. (2013). Experiences of participants in Minifiddlers' distance learning environment. Problems in Music Pedagogy, 12 (2), 93-105.

Soteras, I. (2013). Comparació de tres mètodes per a violí: Shinichi Suzuki, Paul Rolland y Mimi Zweig (Tesis de grado). Recuperada de: http://www.recercat.cat/handle/2072/217011

Starr, W. (1976). The Suzuki Violinist: a Guide for Teachers and Parents. Tenesse: Kingston Ellis Press.

Suzuki, S. (1959). Suzuki violin school: Violin part. Princeton: Summy-Birchard.

Suzuki, S. (1969). Nurtured by Love. New York: Exposition Press.

Szilvay, G. (1996). About Colourstings in General. The East-Helsinki Music Institute. Recuperado de http//www.ihmo.fi/strings/sivutenglanniksi/ CSarticles02.htm

Szilvay, G. (2005). Violin ABC: Book A, Book B, Book C. Tampere: Tammer-Paino Oy.

Szilvay, G. (2015). Colourstrings. Helsinki. Recuperado de http://www.colourstrings.fi

Voima, N. (2009). Child friendly approach to instrumental education, The Colourstrings Violin School (Tesis de grado). Recuperada de: http://www.theseus.fi/bitstream/handle/10024/7246/voima noora.pdf?sequence=1

Willems, E. (1981). El valor humano de la educación musical. Barcelona: Paidós.

(C) LUISA MOYA PASTOR. THE CONTENT OF THIS ARTICLE IS THE SOLE RESPONSIBILITY OF THE AUTHORS. THE REVISTA ELECTRÓNICA DE LEEME AND UNIVERSITAT DE VALĖNCIA ARE NOT LIABLE FOR ANY LEGAL ACTIONS THAT MAY ARISE INVOLVING THE ARTICLE'S CONTENT. REVISTA ELECTRÓNICA DE LEEME -LISTA ELECTRÓNICA EUROPEA DE MÚSICA EN LA EDUCACIÓN-HTTP://MUSICA.REDIRIS.ES.ISSN: 1575-9563 EDITORES: UNIVERSIDAD DE VALENCIA Y JESÚS TEJADA GIMÉNEZ. VISIBILIDAD DE ESTA REVISTA: EBSCO, CINDOC (CESIC), CITEFACTOR, COPAC, DIALNET, DICE (CSIC), DOAJ, E-REVISTAS (CSIC), EBSCO PREMIER, ERIH+, GALE CENGAGE LEARNING, IN-RECS, IRESIE, LATINDEX, MIAR, OCLC WORLDCAT, RESH, REDIB, RILM CORE JOURNALS, SUDOC, ULRICHS, ESTA REVISTA ESTÁ PUBLICADA CON EL APOYO INSTITUCIONAL DE REDIRIS-CONSEJO SUPERIOR DE INVESTIGACIONES CIENTIFICAS Y ES DE ACCESO LIBRE. CREATIVE COMMONS LICENSE 4.0 BY 